\title{
Comparação entre a Histerossonografia, a Histeroscopia e a Histopatologia na Avaliação da Cavidade Uterina de Mulheres na Pós-menopausa
}

\author{
Comparison between Hysterosonography, Hysteroscopy and Histopathology \\ in the Evaluation of Postmenopausal Woman Uterine Cavity \\ Alfeu Cornélio Accorsi Neto, Wagner José Gonçalves, Sérgio Nicolau Mancini \\ José Maria Soares Jr, Mauro Abi Haidar, Geraldo Rodrigues de Lima, Edmund Chada Baracat
}

\begin{abstract}
RESUM0
Objetivo: comparar os resultados da histero-sonografia com os da histeroscopia e com os da histopatologia em mulheres na pós-menopausa.

Métodos: realizou-se histerossonografia, histeroscopia e biópsia endometrial dirigida em 58 mulheres que tinham eco endometrial maior que $4 \mathrm{~mm}$, idade superior a 40 anos, tempo de amenorréia superior a um ano e niveis de hormônio foliculo-estimulante maiores que 35 $\mathrm{mUI} / \mathrm{mL}$. Foram excluidas as pacientes que fizeram uso de hormonioterapia, assim como aquelas com fatores que impedissem a realização dos exames de histerossonografia, histeroscopia ou o estudo histopatológico. A análise estatística foi obtida pelos testes $G$ de Cochran e McNemar. Foram calculados os valores de sensibilidade, especificidade e preditivos positivo e negativo da histero-sonografia comparados com os outros exames.

Resultados: as taxas de concordância da histerossonografia comparada aos achados histerpscópicos e histológicos foram de 94,8 e 77,6\%, respectivamente. A sensibilidade e a especificidade na detecção de anormalidade da cavidade endometrial da histerossonografia comparadas com a histeroscopia foram de 98 e de 75\%, respectivamente. Já os valores preditivos positivo e negativo da hístero-sonografia foram de 96 e de 86\%, respectivamente. Na comparação ao estudo histopatológico para o diagnóstico de alterações da cavidade endometrial, a histerossonografia revelou sensibilidade de $98 \%$, especificidade de 33\% e os valores preditivos positivo e negativo foram de 76 e de $86 \%$, respectivamente. Ressalta-se que das onze pacientes com sinéquia uterina diagnosticada pela histerossonografia, duas tiveram hiperplasia endometrial. Houve um caso de cavidade normal pela histero-sonografia em que o resultado da biópsia foi hiperplasia endometrial simples.

Conclusões: nossos dados sugerem que a histerossonografia tem boa sensibilidade comparada com a histeroscopia. Contudo, há limitações deste método diagnóstico comparado com o histopatológico, principalmente nos casos de sinéquia uterina.
\end{abstract}

PALAVRAS-CHAVE: Pós-menopausa. Cavidade endometrial. Histopatologia. Histeroscopia e histerossonografia.

Departamento de Ginecologia e Obstetrícia da Faculdade de Medicina de Catanduva e Departamento de Ginecologia da Universidade Federal de São Paulo- Escola Paulista de Medicina (UNIFESP-EPM).

Correspondência:

Alfeu Cornélio Accorsi Neto

Rua Recife, 195 - Centro

15800-240 - Catanduva - SP

Fone: (17) 522-7080 - Fax: (17) 521-4561

e-mail: inst.accorsi@catanduva.com.br
Introdução

A menopausa, ou seja, a última menstruação, ocorre, em geral, aos 50 anos $^{1}$. Com a maior longevidade, as mulheres podem viver um terço de suas vidas na pós-menopausa. Conseqüentemente, estão sujeitas às afecções próprias desta fase. Entre elas, se destacam as neoplasias ma- 
lignas dos órgãos genitais, principalmente o câncer do endométrio, cuja freqüência varia com a idade, o estado nutricional, o número de gestações e o uso de estrogênios ${ }^{2}$.

Atualmente, o controle periódico da cavidade uterina e a detecção de alterações endometriais são indispensáveis para o acompanhamento de mulheres em uso de reposição hormonal na pósmenopausa. Entre os métodos mais empregados, destaca-se a ultra-sonografia ${ }^{3,4}$.

Os estudos de Hall et al. ${ }^{5}$ foram pioneiros na investigação morfológica do útero pela ultrasonografia e deram grande impulso para a divulgação da ultra-sonografia uterina. Entretanto, o aprimoramento das técnicas para melhor visualização das alterações endometriais ocorreu no final da década de 80, com o advento dos transdutores transvaginais. Vários trabalhos, entre eles o de Fleischer et al. ${ }^{6}$, mostraram a importância da espessura endometrial aferida pelo eco endometrial para a identificação de afecções endometriais.

Consideram-se como espessura normal do eco endometrial na pós-menopausa valores de até $4 \mathrm{~mm}$; entre 4 e $7 \mathrm{~mm}$ podem-se observar endométrios não atróficos (proliferativo e hiperplásico) e a neoplasia maligna de endométrio com espessura endometrial foi maior que $8 \mathrm{~mm}^{7}$. Assim, quando a espessura do eco endometrial não for sugestiva de atrofia, isto é, for superior a $4 \mathrm{~mm}$, recomenda-se proceder ao estudo histopatológico da mucosa. Atualmente, prefere-se a histeroscopia com biópsia dirigida ${ }^{7}$. Do mesmo modo, outros autores preconizam também a investigação da cavidade uterina quando o eco endometrial ultrapassa 4 ou $5 \mathrm{~mm}^{8,9}$.

Com o propósito de melhorar a imagem da cavidade uterina, Richman et al. ${ }^{10}$ propuseram a instilação por meio de uma cânula rígida, de uma solução de dextran 70 na cavidade uterina, durante a ultra-sonografia transvaginal. Esta técnica foi denominada de histerossonografia. Após a infusão de solução salina na cavidade uterina, Deichert et al. ${ }^{11}$ também confirmaram a superioridade deste exame em relação à ultra-sonografia convencional devido à melhor qualidade da imagem ultra-sonográfica.

Desde então, outros estudos mostraram a simplicidade, tolerabilidade e facilidade da hísterossonografia utilizando cateter flexível e solução salina no diagnóstico de pólipos, miomas e sinéquias, diferenciando-os tanto das hiperplasias quanto das verdadeiras neoplasias ${ }^{12-14}$. Recente pesquisa mostrou que a hísterossonografia é tão eficiente no diagnóstico de leiomioma submucoso quanto a histeroscopia, com a vantagem de ser bem tolerada ${ }^{15}$. Já em pacientes com sangramento da pós-menopausa, o método seria superior à ultra-sonografia transvaginal convencional no diagnóstico de anomalias endometriais ${ }^{14}$.

Assim, o objetivo deste estudo é avaliar a hísterossonografia na investigação da cavidade uterina de mulheres na pós-menopausa com eco endometrial superior a $4 \mathrm{~mm}$, tendo a histeroscopia e a biópsia endometrial como padrão-ouro.

\section{Pacientes e Métodos}

Realizou-se estudo transversal, no qual foram avaliadas 58 pacientes na pós-menopausa, atendidas no Ambulatório de Climatério do Departamento de Ginecologia e Obstetrícia da Faculdade de Medicina de Catanduva e no Setor de Oncocirurgia do Departamento de Ginecologia da Universidade Federal de São Paulo - Escola Paulista de Medicina (UNIFESP-EPM).

Foram incluidas mulheres com sintomas climatéricos, com mais de 40 anos, tempo de amenorréia superior a um ano e níveis de hormônio folículo-estimulante (FSH) superiores a $35 \mathrm{mUI} / \mathrm{mL}$. Foram excluídas as pacientes que fizeram uso de hormonioterapia, assim como aquelas com fatores que impedissem a realização da ultra-sonografia transvaginal, hísterosonografia, histeroscopia ou a coleta de material da cavidade uterina para estudo histopatológico. Após o exame ultra-sonográfico transvaginal, as pacientes com eco endometrial menor que $4 \mathrm{~mm}$ não foram selecionadas para o estudo.

O estudo foi previamente submetido e aprovado pela Comissão de Ética da UNIFESP- EPM. Todas as pacientes assinaram termo de consentimento pós-informado.

Em todas as pacientes foram efetuados anamnese, exames fisico geral e ginecológico e ultrasonografia transvaginal. As que apresentavam eco endometrial maior que $4 \mathrm{~mm}$ foram selecionadas para serem submetidas à hísterossonografia.

Para tanto, as pacientes permaneciam na sala de exame ultra-sonográfico em posição ginecológica, introduzia-se o espéculo de Collins e identificava-se o colo do útero. Efetuava-se a anti-sepsia da vagina e do colo com solução de iodopovidona e, logo em seguida, colocava-se a sonda ZUI 2.0 no interior da cavidade uterina, sem a necessidade de se proceder à dilatação cervical. O balão da sonda era então insuflado e o espéculo vaginal retirado, com posterior introdução do transdutor vaginal. Fazia-se o enchimento gradativo da cavidade uterina com solução salina a $0,9 \%$ até o volume máximo de $10 \mathrm{~mL}$. Concomitantemente, realizava-se a ultra-sonografia transvaginal. 
Considerou-se a imagem da hísterosonografia normal quando o eco endometrial era menor ou igual a $4 \mathrm{~mm}$, e anormal, quando era maior que $4 \mathrm{~mm}$. Nesta eventualidade, o espessamento ou as alterações detectadas poderiam ser localizados ou difusos.

Caracterizou-se o espessamento como difuso quando o eco endometrial, regular ou irregular, com ecotextura homogênea ou heterogênea, comprometia toda a extensão da cavidade uterina. Foram consideradas também as imagens que projetavam-se discretamente para a luz uterina, com ecotextura hiper ou isoecóicas, com limites regulares ou não, sem porém comprometer toda a extensão da cavidade uterina.

Definiu-se o pólipo endometrial como imagem hiperecóica, com limites regulares e precisos, projetando-se para a luz da cavidade uterina. O leiomioma uterino submucoso foi caracterizado por áreas mais hipoecóicas que os pólipos, normalmente isoecóicas com o miométrio, de limites bem definidos. Já as sinéquias apresentavamse como imagens sólidas, pequenas e bem ecogênicas, localizadas na parede endometrial, e que não promoviam sombra acústica.

Para a análise estatística, dividimos a hístero-sonografia em normal quando o eco endometrial era homogêneo, sem qualquer anormalidade intracavitária visivel. O exame foi anormal quando o eco endometrial homogêneo ou heterogêneo apresentava anormalidades intracavitárias. Os resultados da hísterossonografia foram comparados com os da histeroscopia e do exame histopatológico, métodos definidos como padrãoouro para a avaliação de anomalias endocavitárias e endometriais.

De igual modo, para a análise estatística considerou-se a histeroscopia normal quando os achados eram compativeis com atrofia endometrial na ausência de qualquer anormalidade intracavitária, e anormal, quando havia alteração da cavidade uterina (sinéquia ou pólipo) ou exame histopatológico indicativo de proliferação endometrial (endométrio proliferativo, hiperplásico ou neoplásico). Com relação à avaliação histopatológica do endométrio, considerou-se normal quando era atrófico. Todos os demais achados foram referidos como anormais.

Foram utilizados para a análise dos resultados, testes não paramétricos, levando-se em consideração a natureza dos valores das variáveis estudadas. Aplicaram-se os seguintes testes: teste $\mathrm{G}$ de Cochran para estudar a concordância do diagnóstico entre os exames realizados em cada uma das mulheres; teste de McNemar para a comparação dos resultados histopatológicos com o dos diferentes exames (histeroscopia, hísterossonografia). Procedeu-se também ao cálculo da sensibilidade, especificidade e dos valores preditivos positivo e negativo da histeroscopia e da hísterosonografia, tendo-se como padrão-ouro os resultados dos exames histopatológicos. Fixou-se, em todos os testes, o valor de 0,05 ou $5 \%$ para o nível de rejeição da hipótese de nulidade.

\section{Resultados}

Nos 58 exames realizados, a hísterossonografia detectou alterações intracavitárias em 51 (88\%); em 7 casos $(12 \%)$ não mostrou alterações. O diagnóstico, em ordem decrescente de freqüência, foi: pólipo em 26 casos (51\%), sinéquia em 11 (21\%), leiomioma uterino submucoso em 6 (12\%), espessamento endometrial regular em $6(12 \%)$ e espessamento endometrial irregular em 2 (4\%).

Em relação ao exame histeroscópico, nos 58 casos estudados, detectaram-se alterações em 50 $(86 \%)$ e, em $8(14 \%)$, a cavidade uterina mostrouse normal e o endométrio atrófico.

Nos exames histeroscópicos com alterações, os achados mais freqüentes foram pólipo em 29 mulheres (58\%) e sinéquia em 12 (24\%). Em 4 (8\%) observou-se espessamento endometrial, sugestivo de endométrio proliferativo, e em outros $3(6 \%)$, o aspecto foi de leiomioma submucoso. Nas duas restantes $(4 \%)$, havia espessamento endometrial irregular, com hipervascularização, sugestivo de câncer de endométrio.

Em relação às 58 biópsias realizadas, 40 (69\%) mostraram-se alteradas. Encontrou-se pólipo endometrial em $30(52 \%)$ pacientes, hiperplasia sem atipias em $5(9 \%)$, mioma em $2(3 \%)$, adenocarcinoma do endométrio em $2(3 \%)$ e endométrio proliferativo em $1(2 \%)$.

Procurou-se comparar os exames realizados entre si, para aquilatar a concordância entre eles (Tabela 1). Na correlação da hísterossonografia com a histeroscopia, o teste de McNemar não foi significante $(p=0,37)$. A taxa de concordância entre os resultados anormais e normais foi de $94,8 \%$. $\mathrm{Na}$ Tabela 2, os resultados da hísterossonografia foram correlacionados com o resultado histopatológico e verificou-se haver discordância significativa entre os testes $(p=0,003)$, principalmente nos achados anormais da hístero-sonografia. Dos 51 casos que foram detectados como anormais, doze foram normais no estudo histopatológico. A taxa de concordância foi $77,6 \%$. Houve um caso que foi registrado como normal pela hístero-sonografia mas cujo exame histopatológico detectou hiper- 
plasia endometrial simples sem atipia. Na Tabela 3 , foram comparados os dados da histeroscopia com os da histopatologia, sendo que houve discordância em 10 casos de achados anormais pela histeroscopia que não foram confirmados pelo estudo histopatológico $(\mathrm{p}=0,03)$. A taxa de concordância foi de $82,8 \%$.

Tabela 1 - Comparação dos resultados de histero-sonografia e histeroscopia em 58 mulheres na pós-menopausa.

\begin{tabular}{lccr} 
Histerossonografia & \multicolumn{3}{c}{ Histeroscopia } \\
& Anormal & Normal & Total \\
\hline Anormal & 49 & 2 & 51 \\
Normal & 1 & 6 & 7 \\
Total & 50 & 8 & 58 \\
Teste de McNemar $(p=0,37)$ & Taxa de concordância $(94,8 \%)$ \\
\hline
\end{tabular}

Tabela 2 - Comparação dos resultados de histero-sonografia e histopatologia em 58 mulheres na pós-menopausa.

\begin{tabular}{lccr} 
Histerossonografia & \multicolumn{3}{c}{ Histopatologia } \\
& Anormal & Normal & Total \\
\hline Anormal & 39 & 12 & 51 \\
Normal & 1 & 6 & 7 \\
Total & 40 & 18 & 58 \\
teste de McNemar $(\mathrm{p}=0,003)$ & Taxa de concordância $(77,6 \%)$ \\
\hline
\end{tabular}

Tabela 3 - Comparação dos resultados de histeroscopia e histopatologia em 58 mulheres na pós-menopausa.

\begin{tabular}{lccr} 
Histeroscopia & \multicolumn{3}{c}{ Histopatologia } \\
& Anormal & Normal & Total \\
\hline Anormal & 49 & 10 & 51 \\
Normal & 0 & 8 & 7 \\
Total & 40 & 18 & 58 \\
Teste de McNemar $(\mathrm{p}=0,03)$ & Taxa de concordância $(82,8 \%)$ \\
\hline
\end{tabular}

Em seguida, determinaram-se a sensibilidade, a especificidade e os valores preditivos positivo e negativo da hísterossonografia para o diagnóstico de anomalias da cavidade, considerandose a histeroscopia como padrão-ouro. A sensibilidade e a especificidade foram de 98 e de $75 \%$, respectivamente. Em relação ao valor preditivo positivo, definido como sendo a porcentagem de mulheres com alterações intracavitárias quando a hísterossonografia mostrou alteração, foi de $96 \%$. Já o valor preditivo negativo, ou seja, a porcentagem de mulheres nas quais a cavidade não revelara alterações quando a hísterossonografia era normal, foi de $86 \%$.
No entanto, quando se utilizou o estudo histopatológico como padrão-ouro para comparar o diagnóstico de anormalidade da cavidade endometrial, a hísterossonografia revelou sensibilidade de $98 \%$ e especificidade de $33 \%$, com valores preditivos positivo e negativo de 76 e de $86 \%$, respectivamente.

Apesar de a especificidade ser elevada quando comparamos a hísterossonografia com a histeroscopia, detectamos divergência com o exame histopatológico, nos casos de sinéquias uterinas. Nestes, a especificidade do exame foi baixa e foram diagnosticados casos de hiperplasia glandular.

Nos casos com avaliação hístero-sonográfica anormal, ou seja, com alteração endometrial ou intracavitária, observamos em 26 (51\%) imagens sugestivas de pólipo endometrial. Destes, 25 (96\%) foram confirmados pelo estudo histopatológico e um caso (4\%) não foi ratificado, sendo que o diagnóstico histopatológico foi de endométrico atrófico.

Em 11 casos (22\%) fez-se o diagnóstico de sinéquia uterina. Destes, em $8(73 \%)$ o exame histopatológico mostrou endométrio atrófico, em $2(18 \%)$ hiperplasia glandular sem atipia e em um $(9 \%)$ endométrio proliferativo.

Em seis $(12 \%)$ casos com hísterossonografia sugestiva de leiomioma submucoso, o estudo histopatológico confirmou este achado em 2 (33\%). Nos outros $4(67 \%)$, a histopatologia revelou $1(25 \%)$ pólipo, $2(50 \%)$ endométrios atróficos e $1(25 \%)$ hiperplasia glandular sem atipia.

Das seis $(12 \%)$ pacientes com imagem de espessamento endometrial irregular, em 2 (33\%) o resultado histopatológico evidenciou adenocarcinoma, e nas outras 4 (67\%), pólipo endometrial. Em outras duas (4\%), o espessamento era difuso e regular, mas o exame histopatológico revelou tratar-se de endométrio atrófico e hiperplasia glandular sem atipia.

\section{Discussão}

Com o aumento da longevidade e em decorrência da reposição hormonal ser empregada na pós-menopausa para amenizar as repercussões clínicas do hipoestrogenismo, o estudo do endométrio de mulheres nessa fase tem sido motivo de inúmeras pesquisas, visando o rastreamento de lesões precursoras do câncer do endométrio ${ }^{7,16}$. Para tanto novos métodos tem sido aplicados, como a histeroscopia e a histero-sonografia.

Diversos autores relataram a simplicidade, a tolerabilidade e a facilidade de diagnosticar 
pólipos, leiomiomas e sinéquias, diferenciando-os dos espessamentos difusos como as hiperplasias e neoplasias, usando a histerosonografia ${ }^{14-18}$. Estes trabalhos mostram que a a histerossonografia é bom método para rastreamento de alterações da cavidade endometrial. Nossos resultados confirmam os dados da literatura, mas este método ainda apresenta algumas limitações quando o seu resultado é normal: um caso de hiperplasia endometrial simples sem atipia foi diagnósticado como normal na histerossonografia.

A sensibilidade da histerossonografia quando comparada a histeroscopia foi alta, detectando anormalidades na cavidade uterina em 98\% das pacientes com histeroscopia anormal. Este fato sugere que este método é muito sensivel para o rastreamento e o diagnóstico as alterações endometriais. Kazandi et al. ${ }^{14}$ obtiveram resultados semelhantes, entretanto, as mulheres estudadas tinham o eco endometrial maior $4 \mathrm{~mm}$ e apresentaram episódios de sangramento uterino da pós-menopausa, ao passo que as nossas pacientes não relataram esta queixa. Wolman et al. ${ }^{19} \mathrm{en}-$ contraram tanto sensibilidade quanto especificidade elevadas quando compararam os resultados da histero-sonografia com a histeroscopia.

Apesar da especificidade ser elevada quando comparamos a histerossonografia com a histeroscopia, detectamos divergência com o exame histopatológico, nos casos de sinéquias uterinas. Nestes, a especificidade do exame foi baixa e foram diagnosticados casos de hiperplasia glandular. Esta parece a limitação deste exame. A explicação para falha do exame seria a dificuldade de expandir a cavidade endometrial e obter imagens mais representativas das alterações endometriais em processo.

Em nosso estudo, na avaliação de pacientes na pós-menopausa sem queixas de sangramento genital com imagens anormais na cavidade uterina detectadas pela ultra-songrafia, a histerossonografia mostrou-se ser recurso fácil, de baixo custo e indolor. É ainda capaz de elucidar dúvidas na imagem ultra-sonográfica transvaginal, ao aprimorar o diagnóstico. Distinguimos, de maneira bastante clara, os pólipos, os leiomiomas e as sinéquias das hiperplasias e neoplasias malignas. A histerossonografia selecionou, com maior sensibilidade, as pacientes que realmente necessitaram de investigação histopatológica do endométrio orientada e/ou dirigida por métodos mais invasivos, como a histeroscopia.

Nossos dados sugerem que a histerossonografia apresenta boa sensibilidade comparada a histeroscopia. Contudo, há limitações deste método diagnóstico comparado com o histopatógico, principalmente nos casos de sinéquia uterina.

\section{ABSTRACT}

Purpose: to compare the results of hysterosonography with those of hysteroscopy and the histopathologic study in postmenopausal women.

Methods: hysterosonography, hysteroscopy and endometrial biopsy were performed in 59 women who had an endometrial echo over $4 \mathrm{~mm}$, age above 40 years and amenorrhea over one year, and whose follicle-stimulating hormone levels were over $35 \mathrm{mIU} / \mathrm{mL}$. Patients using hormones were excluded, as well those in whom it was impossible to perform histerosonography, histeroscopy or endometrial biopsy. The statistical analysis was performed using the nonparametric "G"-Cochran and McNemar tests. In addition, sensitivity and specificity, as well as positive and negative predictive values were determined. The value of 0.05 or $5 \%$ for rejection level of the null hypothesis was applied.

Results: the agreement rates of hysterosonographic results compared to hysteroscopy and histopatology were 94.8 ande $77.6 \%$, respectively. Sensitivity and specificity of hysterosonographic evaluation of the abnormal endometrial cavity were 98 and 75\%, respectively, when compared to hysteroscopy. In addittion, positive and negative predictive values of hysterosonography were 96 and $86 \%$, respectively. When the histopathologic study was used as the gold standard, sensitivity and specificity were 98 and 33\%, with positive predictive value of $76 \%$ and negative predictive value of $86 \%$, for the detection of the endometrial cavitary changes. One great concern were the histopathologic results of two patients with uterine synechia who showed endometrial hyperplasia. Also, one patient was diagnosed as normal using histerosonography and the histopatological result showed simple hyperplasia.

Conclusion: our data suggest that hysterosonography presented good sensitivity as compared with hysteroscopy. However, uterine synechia is the great limitation of this method as compared with histopathology.

KEYWORDS: Postmenopausal uterine cavity. Histopathology. Hysteroscopy and hysterosonography.

\section{Referências}

1. Baracat EC, Soares Júnior JM, Haidar MA, Rodrigues de Lima G. Aspectos reprodutivos no climatério. In: Fernandes CE, editor. Menopausa: diagnóstico e tratamento. $1^{\text {a }}$ ed. São Paulo: Segmento; 2003, p.125-9.

2. Kaaks R, Lukanova A, Kurzer MS. Obesity, endogenous hormones, and endometrial cancer risk: a synthetic review. Cancer Epidemiol Biomarkers Prev 2002; 11:1531-43.

3. Gull B, Karlsson B, Milsom I, Granberg S. Can ultrasound replace dilation and curettage? A 
longitudinal evaluation of postmenopausal bleeding and transvaginal sonographic measurement of the endometrium as predictors of endometrial cancer. Am J Obstet Gynecol 2003; 188:401-8.

4. Davidson KG, Dubinsky TJ. Ultrasonographic evaluation of the endometrium in postmenopausal vaginal bleeding. Radiol Clin North Am 2003; 41:769-80.

5. Hall DA, Hann LE, Ferrucci JT Jr, et al. Sonographic morphology of the normal menstrual cycle. Radiology 1979; 133:185-8.

6. Fleischer AC, Kalemeris GC, Machin JE, Entman SS, James AE Jr. Sonographic depiction of normal and abnormal endometrium with histopathologic correlation. J Ultrasound Med 1986; 5:445-52.

7. Rodrigues de Lima G, Gonçalves WJ, Nicolau, SM, Bortoletto CCR. Neoplasias malignas do endométrio. In: Borges RD, Rothschild HA, editores. Atualização Terapêutica. $21^{\mathrm{a}}$ ed. São Paulo: Artes Médicas; 2003. p.660-4.

8. Bakour SH, Dwarakanath LS, Khan KS, Newton JR, Gupta JK. The diagnostic accuracy of ultrasound scan in predicting endometrial hyperplasia and cancer in postmenopausal bleeding. Acta Obstet Gynecol Scand 1999; 78:447-51.

9. Bronz L, Dreher E, Almendral A, Studer A, Haller U. Guideline for the diagnosis of postmenopausal bleeding. PMPB Working Group of the SGGG. Gynakol Geburtshilfliche Rundsch 2000; 40:71-9.

10.Richman TS, Viscomi GN, deCherney A, Polan ML, Alcebo LO. Fallopian tubal patency assessed by ultrasound following fluid injection. Work in progress. Radiology 1984; 152:507-10.

11.Deichert U, van de Sandt M, Lauth G, et al. Vaginale Hysterokontrast-sonographie zur differential- diagnostischen Abklärung eines Pseudgestationssackes. Ultraschall Klin Prax 1987; 2:245-8.

12.Tepper R, Beyth Y, Altaras MM, et al. Value of sonohysterography in asymptomatic postmenopausal tamoxifen-treated patients. Gynecol Oncol 1997; 64:386-91.

13.Bonilla-Musoles F, Raga F, Osborne NG, Blanes J, Coelho F. Three-dimensional hysterosonography for the study of endometrial tumors: comparison with conventional transvaginal sonography, hysterosalpingography, and hysteroscopy. Gynecol Oncol 1997; 65:245-52.

14.Kazandi M, Aksehirli S, Cirpan T, Akercan F. Transvaginal sonography combined with saline contrast sonohysterography to evaluate the uterine cavity in patients with abnormal uterine bleeding and postmenopausal endometrium more than $5 \mathrm{~mm}$. Eur J Gynaecol Oncol 2003; 24:185-90.

15.Leone FP, Lanzani C, Ferrazzi E. Use of strict sonohysterographic methods for preoperative assessment of submucous myomas. Fertil Steril 2003; 79:998-1002.

16.Alves de Lima FO, Vasserman J, Baracat EC. Aspecto histológico do endométrio na pós-menopausa. J Bras Ginecol 1979; 87:303-19.

17.Parsons AK, Lense JJ. Sonohysterography for endometrial abnormalities: preliminary results. J Clin Ultrasound 1993; 21:87-95.

18.Goldstein SR. Saline infusion sonohysterography. Clin Obstet Gynecol 1996, 39:248-58.

19.Wolman I, Jaffa AJ, Hartoov J, Bar-Am A, David MP. Sensitivity and specificity of sonohysterography for the evaluation of the uterine cavity in perimenopausal patients. J Ultrasound Med 1996; 15:285-8.

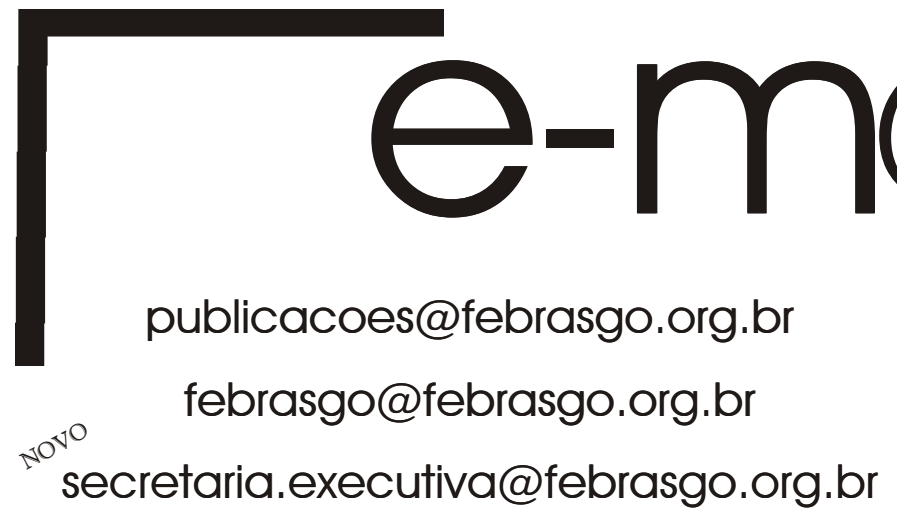

$$
\begin{aligned}
& \text { № presidencia@febrasgo.org.br } \\
& \text { 1000 habilitacao@febrasgo.org.br } \\
& \text { तNo } \\
& \text { tego@febrasgo.org.br }
\end{aligned}
$$

\section{DÚVIDAS - SUGESTÕES - ESCLARECIMENTOS MANDE SEU E-MAIL}

